\title{
Opportunities to enhance perioperative patient safety: 2013 and beyond
}

\author{
Donald R. Miller, MD
}

Published online: 12 December 2012

(c) Canadian Anesthesiologists' Society 2012

We are pleased to dedicate this special theme issue of the Journal to the important topic of perioperative patient safety. Anesthesiologists take justifiable pride in the development and implementation of robust patient safety initiatives throughout the world. At the same time, many of us are challenged daily in caring for patients with everincreasing medical complexity. We provide anesthesia for routine and complex surgical and interventional procedures in the operating room and "remote settings" within the context of an ever-complex working environment. In considering these contemporary and patient-focused challenges, some months ago, our editorial team invited experts from Canada and internationally to address current key issues in perioperative patient safety in a series of brief review articles. I acknowledge with special thanks our Board Member, Dr. Alan F. Merry, for his thoughtful input and contribution to this issue, and for his instrumental role as co-editor and editorialist. ${ }^{1}$ According to Journal policy, these articles have all been externally reviewed and edited, and for the benefit of our readers, we have harmonized the outline of each article to include a structured abstract, a list of key words, and two or three multiple choice questions to guide readers' attention to the key points.

In the first review article, Dr. John H. Eichhorn provides an overview of practical current issues in perioperative patient safety. He emphasizes the fact that important gains in patient safety over the past three decades have been attributed largely to the development of behaviours known as "safety monitoring". ${ }^{2}$ Extension of the human senses

\section{R. Miller, MD $(\bowtie)$}

Department of Anesthesia, The Ottawa Hospital and University

of Ottawa, General Campus CCW 1401, 501 Smyth Road,

Ottawa, ON K1H 8L6, Canada

e-mail: dmiller@ottawahospital.on.ca through electronic monitors, such as capnography and pulse oximetry, has been a major advance, but Dr. Eichhorn cautions that, "in the highly developed world, this current success is threatened by complacency and production pressure" which bring forth new challenges.

Electronic medical records, hence, anesthesia information management systems (AIMS), provide enormous potential to enhance patient safety. Anesthesiologists have the opportunity to become key leaders in the adoption, implementation, and integration of AIMS and healthsystem information technology (IT) solutions in general. In their review article on the evolving role of IT in perioperative patient safety, Drs. Michael Stabile and Lebron Cooper identify that uptake of health IT and AIMS remains variable despite the potential benefits, and they identify advantages and barriers to the adoption and implementation of IT in general and AIMS in particular. ${ }^{3}$

Later articles in this theme issue address specific clinical issues that present ongoing challenges to the practicing clinician. Medication error remains a leading cause of adverse events in the perioperative setting. In their article "Improving drug safety for patients undergoing anesthesia and surgery," Drs. Beverley A. Orser, Sylvia Hyland, C. Ruth Wilson, David U, and Ian Sheppard summarize recent advances, tools and interventions designed to improve drug safety, relevant to the practicing anesthesiologist. ${ }^{4}$ Important Canadian and international initiatives such as new standards for labelling of drug ampoules and vials, and the development of bar code systems that provide as systematic approach to "double checking" or drug verification in the operating room, are reviewed. This article also addresses the important subject of medication reconciliation as a means to enhance patient safety.

There is now fairly compelling evidence that implementation of checklists into clinical practice can enhance 
patient safety. In their review article on perioperative checklist methodologies, Dr. Thomas G. Weiser and William R. Berry consider some of the mechanisms by which checklists work and evaluate evidence supporting their use. ${ }^{5}$ Another important topic in this theme issue is the role of practice guidelines and evidence-based medicine in perioperative patient safety. In his review article, Dr. Edward Crosby discusses the impact of clinical practice guidelines on outcomes of care and patient safety. "Under certain conditions, guidelines can add value to care and improve outcomes; they need to be evidence-based, methodologically sound, and appropriately applied to patients and clinical scenarios. Simply summarizing evidence in a guideline is an inadequate process. To achieve the benefit of guidelines, implementation strategies need to be robust." 6

We invited Dr. Angela Enright, former President of the World Federation of Societies of Anaesthesiologists (WFSA), to provide an update on safety aspects of anesthesia in under-resourced locations. Dr. Enright identifies that an unresolved shortage of trained anesthesia providers (both physician and non-physician alike), especially outside urban areas, is an important contributing factor to high rates of morbidity and mortality in some developing countries. $^{7}$ There is also a well-recognized shortage of equipment and related resources for anesthesia. Lifebox is one example of a charitable foundation dedicated to supplying pulse oximeters to low- and middle-income countries. The recent adoption of the World Health Organization's Surgical Safety Checklist is a low-cost initiative that can further reduce morbidity and mortality.

Drs. Jilles B. Bijker and Adrian W. Gelb examine the role of hypotension in perioperative stroke. Although hypoperfusion is often identified as a possible cause of postoperative stroke, these experts indicate that the role of intraoperative hypotension in the occurrence and development of postoperative cerebral ischemia has never been the subject of thorough investigation. ${ }^{8}$ In this review article, the authors provide an overview of the available literature on the subject, and they identify challenges to help resolve the problem of perioperative cerebral ischemia.

Drs. Gregory Hare, John Freedman, and C. David Mazer examine other important patient safety issues posed by the risks of anemia and related transfusion strategies in the perioperative setting. Impairment of tissue oxygen delivery likely contributes as a central mechanism to anemiainduced organ injury; however, "the existing treatments for anemia (i.e., transfusion, erythropoiesis stimulating agents, blood substitutes) have not produced a demonstrable improvement in patient outcomes despite their efficacy to increase blood oxygen content." 9 The authors review and examine new strategies to avoid both anemia and transfusion with the goal to improve patient outcomes and safety.
Another practical and topical issue is the anesthesiologist's role in the prevention of surgical site infections (SSIs). Herein, Drs. Shawn S. Forbes and Richard F. McLean consider the important role of anesthesiologists "as champions in the multidisciplinary efforts for the prevention of SSIs". ${ }^{10}$ From a different perspective, the overall theme of perioperative patient safety would be incomplete without addressing various aspects of airway management. In their review article, Drs. Jean-Baptiste Paolini, François Donati, and Pierre Drolet consider whether video laryngoscopy will evolve into another tool for difficult intubation or into a new paradigm for airway management. These authors suggest that the greater effectiveness of video laryngoscopes associated with multiperson visualization could enhance overall patient safety during airway management. At the same time, they caution that routine use of video laryngoscopy introduces issues that need to be addressed to avoid potentially dangerous pitfalls. $^{11}$

Finally, we invited Drs. Viren N. Naik and Susan E. Brien, international experts in medical simulation, to consider and review how simulation can serve as an important means to address and improve patient safety. ${ }^{12}$ This fine review article addresses the role of technical and nontechnical skills in routine and crisis situations, and considers different simulation modalities to address the fundamental skills and competencies required to enhance patient safety.

We encourage readers to self-test their knowledge by reviewing and completing the multiple-choice questions at the end of this issue. The correct responses can be found online as Electronic Supplemental Material. ${ }^{13}$ It is our sincere hope that this theme-based issue of the Journal will stimulate clinical decision making and challenge us to reflect on opportunities to further enhance the safety of the perioperative experience for every patient under our care.

\section{Les occasions d'améliorer la sécurité périopératoire des patients, en 2013 et après}

Nous avons le plaisir de consacrer ce numéro spécial du Journal au thème important de la sécurité périopératoire des patients. Les anesthésiologistes se targuent - avec raison - d'avoir réalisé et mis en œuvre des initiatives solides visant à promouvoir la sécurité des patients de par le monde. En même temps, nous sommes nombreux à faire face quotidiennement à des défis dans les soins à des patients présentant des problèmes toujours plus complexes. Nous fournissons l'anesthésie pour des chirurgies ou des 
interventions, qu'elles soient de routine ou complexes, en salle d'opération ou hors bloc, dans le cadre d'un environnement de travail toujours difficile. Il y a quelques mois, en réfléchissant à ces défis actuels, centrés sur le patient, notre équipe de rédaction a invité des experts du Canada et d'ailleurs à aborder des questions clés d'actualité touchant à la sécurité périopératoire des patients dans une série d'articles de synthèse courts. J'aimerais tout particulièrement remercier le Dr Alan F. Merry, membre de notre Comité, pour ses nombreuses contributions réfléchies à ce numéro, ainsi que pour son rôle prépondérant en tant que co-rédacteur et éditorialiste. ${ }^{1}$ Conformément à la politique du Journal, chaque article a été revu et corrigé par un spécialiste externe. Dans l'intérêt de nos lecteurs, nous avons réorganisé le plan de chaque article de façon à ce qu'il comprenne un résumé structuré, une liste de $\mathrm{m}$ ots-clés, et deux ou trois questions à choix de réponse afin de guider l'attention du lecteur vers les points essentiels.

Dans le premier article de synthèse, le Dr John $\mathrm{H}$. Eichhorn propose une vue d'ensemble des questions pratiques actuelles concernant la sécurité périopératoire des patients. Il souligne le fait que d'importants progrès en matière de sécurité des patients survenus au cours des trente dernières années sont attribuables en grande partie à la mise au point de comportements que l'on nomme « monitorage de sécurité ». ${ }^{2}$ L'extension des sens humains par le biais de moniteurs électroniques tels que la capnographie et l'oxymétrie de pouls constitue un progrès majeur. Toutefois, le Dr Eichhorn nous met en garde : «dans le monde très développé, ce succès actuel est mis en péril par la complaisance et la pression à la production », qui amènent leur lot de nouveaux défis.

Les dossiers médicaux électroniques, et par conséquent les systèmes de gestion des informations de l'anesthésie (AIMS), offrent un potentiel énorme en matière d'amélioration de la sécurité des patients. Les anesthésiologistes ont la possibilité de devenir des chefs de file de l'adoption, de la mise en œuvre et de l'intégration des AIMS et des solutions informatiques pour les soins de santé en général. Dans leur article de synthèse sur le rôle en évolution de l'informatique dans la sécurité périopératoire des patients, les Drs Michael Stabile et Lebron Cooper remarquent que l'adoption de l'informatique pour les soins de santé et des AIMS reste très variable malgré les avantages potentiels de ces systèmes. Ils présentent également les avantages et les barrières à l' adoption et à la mise en œuvre de l'informatique en général et des AIMS en particulier. ${ }^{3}$

Les articles subséquents de ce numéro spécial traitent de problèmes cliniques spécifiques qui présentent des défis constants au clinicien. Les erreurs médicamenteuses demeurent une cause majeure d'effets secondaires indésirables dans le contexte périopératoire. Dans leur article sur « L'amélioration de la sécurité des médicaments pour les patients subissant une anesthésie et une chirurgie », les Drs
Beverley A. Orser, Sylvia Hyland, C. Ruth Wilson, David U et Ian Sheppard résument les progrès, outils et interventions récents conçus pour améliorer la sécurité des médicaments qui sont pertinents pour l'anesthésiologiste en pratique. ${ }^{4}$ D'importantes initiatives canadiennes et internationales sont passées en revue, telles que de nouvelles normes pour l'étiquetage des ampoules et des flacons et la mise au point de système de codes à barres qui permettent une approche systématique de «double vérification » ou de vérification du médicament dans la salle d'opération. Cet article traite également du sujet important de la réconciliation des médicaments en tant que façon d'améliorer la sécurité des patients.

Il existe aujourd'hui des données probantes assez convaincantes soutenant que la mise en œuvre de listes de contrôle dans la pratique clinique peut améliorer la sécurité des patients. Dans leur article de synthèse sur les méthodologies de listes de contrôle périopératoires, les Drs Thomas G. Weiser et William R. Berry analysent certains des mécanismes qui font que les listes de contrôle fonctionnent et évaluent les données probantes recommandant leur utilisation. ${ }^{5}$ Un autre thème important de ce numéro spécial touche aux rôles respectifs des directives de pratique et de la médecine factuelle dans la sécurité périopératoire des patients. Dans son article de synthèse, le Dr Edward Crosby traite de l'impact des directives de pratique clinique sur les pronostics de soins et la sécurité des patients. " Dans certaines situations, les directives ajoutent de la valeur aux soins et améliorent les pronostics; elles doivent être fondées sur des données probantes, rigoureuses d'un point de vue méthodologique et appliquées de façon adaptée aux patients et aux situations cliniques. Il ne suffit pas de résumer des données probantes pour créer une directive; c'est là un processus inadéquat. Pour concrétiser les avantages des directives, les stratégies de mise en œuvre doivent être solides. ${ }^{6}$

Nous avons invité la Dre Angela Enright, ex-présidente de la Fédération mondiale des sociétés d'anesthésiologistes (FMSA), à nous présenter les dernières données concernant les aspects de sécurité de l'anesthésie dans les régions où les ressources sont rares. La Dre Enright note qu'une pénurie non résolue de professionnels formés en anesthésie (qu'il s'agisse de médecins ou de non-médecins), particulièrement en dehors des centres urbains, est un facteur qui contribue de façon significative à des taux élevés de morbidité et de mortalité dans certains pays en voie de développement. ${ }^{7}$ Il existe également une pénurie bien connue de matériel et de ressources pour l'anesthésie. Lifebox est un exemple de fondation caritative qui se consacre à la fourniture d'oxymètres de pouls aux pays à faible et moyen revenu. L'adoption récente de la Liste de contrôle de la sécurité chirurgicale de l'Organisation mondiale de la Santé est une initiative qui pourrait encore plus réduire la morbidité et la mortalité. 
Les Drs Jilles B. Bijker et Adrian W. Gelb examinent le rôle de l'hypotension dans l'accident vasculaire cérébral périopératoire. Bien que l'hypoperfusion soit souvent reconnue comme cause possible d'un accident vasculaire cérébral postopératoire, ces experts indiquent que le rôle de l'hypotension peropératoire dans la survenue et le développement de l'ischémie cérébrale postopératoire n'a jamais fait l'objet de recherches approfondies. ${ }^{8}$ Dans cet article de synthèse, les auteurs proposent une vue d'ensemble de la littérature disponible à ce sujet, et ils identifient les défis afin d'aider à résoudre le problème de l'ischémie cérébrale périopératoire.

Les Drs Gregory Hare, John Freedman et C. David Mazer examinent d'autres questions importantes touchant à la sécurité des patients soulevées par les risques d'anémie ainsi que les stratégies transfusionnelles associées dans le contexte périopératoire. La détérioration de l'apport en oxygène aux tissus joue probablement un rôle central dans les lésions aux organes induites par l'anémie; toutefois, « les traitements existants pour l'anémie (c.à-d. la transfusion, les agents stimulateurs de l'érythropoïèse, les substituts sanguins) n'ont pas entraîné d'amélioration démontrable dans les pronostics des patients, malgré leur efficacité à augmenter le contenu d'oxygène dans le sang. $\gg{ }^{9}$ Les auteurs passent en revue et examinent de nouvelles stratégies destinées à éviter l'anémie et la transfusion, avec pour objectif d'améliorer les pronostics et la sécurité des patients.

Le rôle de l'anesthésiologiste dans la prévention des infections du site opératoire (ISO) constitue un autre sujet pratique abordé dans ce numéro spécial. Dans leur article, les Drs Shawn S. Forbes et Richard F. McLean réfléchissent au rôle important des anesthésiologistes « en tant que champions dans les efforts pluridisciplinaires pour la prévention des ISO »..$^{10}$ Aucune discussion globale de la sécurité périopératoire des patients ne serait complète si l'on ne traitait pas aussi des divers aspects de la prise en charge des voies aériennes. Dans leur article de synthèse, les Drs Jean-Baptiste Paolini, François Donati et Pierre Drolet tentent de déterminer si la vidéolaryngoscopie deviendra un autre outil pour l'intubation difficile ou un nouveau paradigme pour la prise en charge des voies aériennes. Ces auteurs suggèrent que la plus grande efficacité des vidéolaryngoscopes, associée à une visualisation par plusieurs personnes, pourrait améliorer la sécurité globale des patients pendant la prise en charge des voies aériennes. Du même souffle, ils nous mettent en garde : l'utilisation routinière de la vidéolaryngoscopie introduit des problèmes et des questions qu'il faut traiter si l'on souhaite éviter des pièges potentiellement dangereux. ${ }^{11}$

Enfin, nous avons invité les Drs Viren N. Naik et Susan E. Brien, deux experts internationaux en matière de simulation médicale, à présenter leurs réflexions sur la place de la simulation comme moyen important d'aborder la sécurité des patients et de l'améliorer. ${ }^{12} \mathrm{Ce}$ bel article de synthèse traite du rôle des compétences techniques et non techniques dans les situations de routine et de crise, et présente différentes modalités de simulation permettant d'acquérir les compétences et habiletés fondamentales nécessaires à l'amélioration de la sécurité des patients.

Nous encourageons nos lecteurs à tester leurs connaissances en passant en revue et en complétant les questions à choix de réponse à la fin de ce numéro. Les bonnes réponses sont disponibles en ligne en matériel électronique supplémentaire. ${ }^{13}$ Nous espérons sincèrement que ce numéro spécial du Journal stimulera la prise de décision clinique et nous mettra au défi de réfléchir aux occasions d'améliorer encore plus la sécurité de l'expérience périopératoire pour chacun des patients dont nous avons la responsabilité.

Competing interests None declared.

\section{References}

1. Merry AF. An overview of quality and safety in health care. Can J Anesth 2013; 60: this issue. DOI: 10.1007/s12630-012-9850-1.

2. Eichhorn JH. Review article: Practical current issues in perioperative patient safety. Can J Anesth 2013; 60: this issue. DOI: 10.1007/s12630-012-9852-z.

3. Stabile M, Cooper L. Review article: The evolving role of information technology in perioperative patient safety. Can J Anesth 2013; 60: this issue. DOI: 10.1007/s12630-012-9851-0.

4. Orser BA, Hyland S, Wilson CR, U D, Sheppard I. Improving drug safety for patients undergoing anesthesia and surgery. Can J Anesth 2013; 60: this issue. DOI: 10.1007/s12630-012-9853-y.

5. Weiser TG, Berry WR. Review article: Perioperative checklist methodologies. Can J Anesth 2013; 60: this issue. DOI: 10.1007/s12630-012-9854-x.

6. Crosby E. Review article: The role of practice guidelines and evidence-based medicine in perioperative patient safety. Can J Anesth 2013; 60: this issue. DOI: 10.1007/s12630-012-9855-9.

7. Enright A. Review article: Safety aspects of anesthesia in underresourced locations. Can J Anesth 2013; 60: this issue. DOI: 10.1007/s12630-012-9856-8.

8. Bijker JB, Gelb AW. Review article: The role of hypotension in perioperative stroke. Can J Anesth 2013; 60: this issue. DOI: 10.1007/s12630-012-9857-7.

9. Hare GM, Freedman J, Mazer CD. Review article: Risks of anemia and related management strategies: can perioperative blood management improve patient safety? Can J Anesth 2013; 60: this issue. DOI: 10.1007/s12630-012-9861-y.

10. Forbes SS, McLean RF. Review article: The anesthesiologist's role in the prevention of surgical site infections. Can J Anesth 2013; 60: this issue. DOI: 10.1007/s12630-012-9858-6.

11. Paolini JB, Donati F, Drolet P. Review article: Video-laryngoscopy: another tool for difficult intubation or a new paradigm in airway management? Can J Anesth 2013; 60: this issue. DOI: 10.1007/s12630-012-9859-5.

12. Naik VN, Brien SE. Review article: Simulation: a means to address and improve patient safety. Can J Anesth 2013; 60: this issue. DOI: 10.1007/s12630-012-9860-z.

13. Multiple Choice Questions. Innovation in perioperative patient safety. Can J Anesth 2013; 60: this issue. DOI: 10.1007/s12630-012-9864-8. 